\title{
MIOCENE BRYOZOANS FROM JABAL ZALTAN, NORTHEAST SIRT BASIN, LIBYA
}

\author{
Yasser A. El-Safori ${ }^{1}$ and Ahmed M. Muftah ${ }^{2}$ \\ ${ }^{1}$ Department of Geology, Faculty of Science, Ain Shams University, P.O. Box 11566 Cairo, Egypt, \\ yasser_elsafori@sci.asu.edu.eg \\ ${ }^{2}$ Department of Earth Sciences, Faculty of Science, University of Benghazi, P. O. Box 9480, Benghazi, \\ Libya. ahmed59muftah@gmail.com
}

\begin{abstract}
A taxonomic study has been performed for the thirty-two retrieve bryozoan species from the measured Serravallian Maradah Formation section at Z138, Jabal Zaltan, northeastern Sirt Basin, Libya. Among this unique assemblage only two new species Calpensia spinosa and Thalamoporella zaltaniensis have been established. The Cubitostrea shells provide the most attachments of the encrusting bryozoans.

The reported species are of Mediterranean/Atlantic distributional affinity. However the new species are considered as an endemic species. The limestones and marls are rich in erect flexible and encrusting forms which indicate inter-subtidal $(10-50 \mathrm{~m})$ environments with moderate rate of sedimentation and relatively strong wave actions. Highly agitated environment is specially recognized from the investigated assemblages in the lower part of Ar Rahlah Member of Maradah Formation as suggested on taphonomical basis.
\end{abstract}

Keywords: Mediterranean, Miocene Bryozoa, Libya, Maradah Formation, Jabal Zaltan

\section{INTRODUCTION}

Jabal Zaltan area is located in the vicinity of the Marada Oasis in north-central Sirt Basin (Longitudes $18^{\circ} 45^{\prime}$ to $20^{\circ} 10^{\prime} \mathrm{E}$ and latitudes $28^{\circ} 20^{\prime}$ to $29^{\circ} 25^{\prime} \mathrm{N}$. The studied locality Z138 is indicated by solid circles (Fig. 1). Maradah Formation (Middle Miocene) was introduced and described by Desio (1935) from 80 meters thick section at Garet al Mazzala, near Marada Oasis. It consists mainly of siliciclastics with minor carbonates at the lower half of the Formation resembling Qarat Jahanam Member changed upwards to mainly carbonate with interbeds of siliciclastics containing marine invertebrates at some levels. This formation is unconformably underlain by the Oligocene Bu Hashisha Formation of Rupelian age, while the upper boundary is not present. According to Mastera (1985) this formation has been subdivided into two members, the lower siliciclastic Qarat Jahannam Member of Burdigallian-Aquitanjan age and the upper carbonate - siliciclastic Ar Rahlah Member (Aquitanian - Serravallian). The type locality of Ar Rahlah Member is situated $25 \mathrm{~km}$ northeast of the Maradah oasis, with about $150 \mathrm{~m}$ thick sequence. Ar Rahlah Member resembles the marine facies with several fossiliferous horizons. It is gradually changed to Qarat Jahannam Member by depth, but unconformably overlain by Wadi Yunis Member of Al Khums Formation (Mastera, 1985). El Hawat (2008) studied the sequence stratigraphy architecture of the Maradah Formation and attributed it to the interplay of steady eustatic sea level change overprinted by spasmodic tectonic activity of Sirt rift complex during Middle Miocene.

\section{LOCATION OF THE Z138 (CAMP SECTION)}

Coordinates: Latitudes ( $\left.28^{\circ} 55^{\prime} 00.5^{\prime \prime} \mathrm{N}\right)$ and Longitudes $\left(19^{\circ} 45^{\prime} 54.6^{\prime \prime} \mathrm{E}\right)$. The measured thickness of this section is about 87 meters, representing Ar Rahlah Member of Maradah Formation (Figs. 1, 2). The section consists mainly of invertebrate-bearing carbonates with minor interbeds of non-fossiliferous siliciclastics. 
El-Safori, Y. A. and Muftah, A. M.

Fig. 1: Location map of Jabal Zaltan area, showing the studied Z138 section.
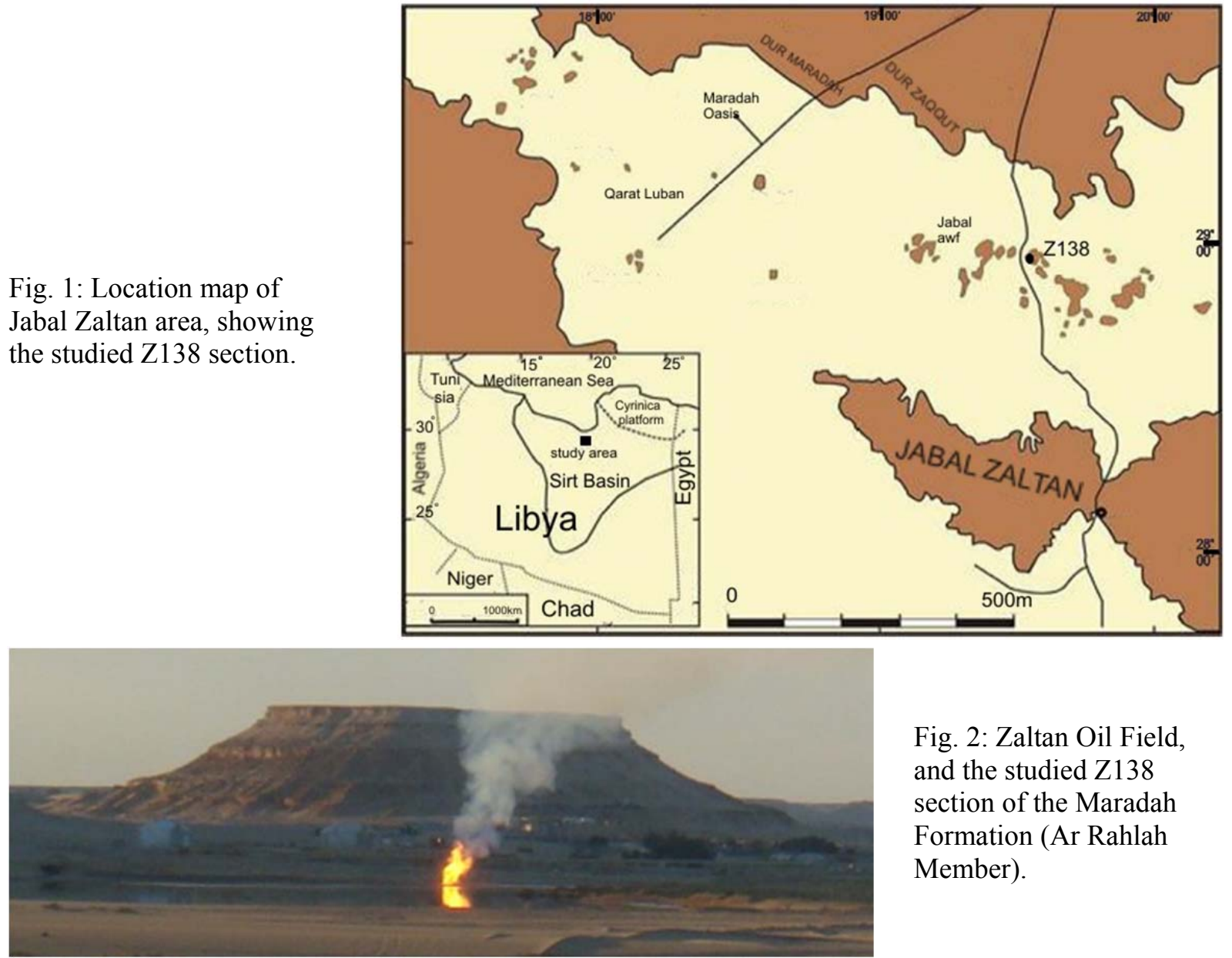

Fig. 2: Zaltan Oil Field, and the studied Z138 section of the Maradah Formation (Ar Rahlah Member).

The raw material of this chapter has been collected during the field trip of the season 2010 by ELNRP team. During which one section (Z138) in Jabal Zaltan area (Figs. 1,2) have been measured, sampled and described. This section mainly represented by the older siliciclastic deposits of the carbonates with siliciclastic alternation of Ar Rahlah Member. Fifty-five samples have been collected from the Z138 at Jabal Zaltan (Figs. 2, 3). Well preserved and abundant bryozoan specimens were picked form the processed microfossils-bearing samples by aid of Kruss stereo zoom binocular microscope, selected species are also examined using a Jeol JSM 6360 Scanning Electron Microsope, at the University of Athens, Department of Historical Geology and Paleontology, for taxonomic and illustrative purposes. All materials (samples and micropaleontological slides) are stored at the micropaleontological section of the Earth Sciences Department of Benghazi University, Benghazi-Libya.

\section{LITHOSTRATIGRAPHY OF AR RAHLAH MEMBER OF MARADAH FORMATION}

Ar Rahlah Member of Maradah Formation at locality Z138 yielded 88m thick sequence, out of which a total of 55 samples have been collected. Neither the upper boundary, nor the lower were observed in this section. The sedimentological details are illustrated in Figure 3. Lithological nature, sedimentary structures, biological contents and ichnofossils are utilized in the recognition of the microfacies and depositional paeoenvironment. The collected samples have been processed petrographically and micropaleontologically, in order to assist in reaching the goals of this study. The studied facies generally reflect gradual changes from siliciclastic dominated intervals to fossiliferous carbonates, with rhythmic sedimentary pattern locally reported. They are formed in response the 
Miocene bryozoans from Jabal Zaltan, Northeast Sirt basin

climatic change during deposition. The highly fossiliferous horizons of mostly bryozoans-bearing sediments are repeatedly reported, indicating low sedimentation rate within the section. The presence of Echinolampas in two major horizons is also recorded. Two distinct hard grounds have also been documented, reflecting eustatic sea level fall accompanied with slow rate of sedimentation.

Fig. 3: Lithological columnar section of Z138 at Jabal Zaltan.

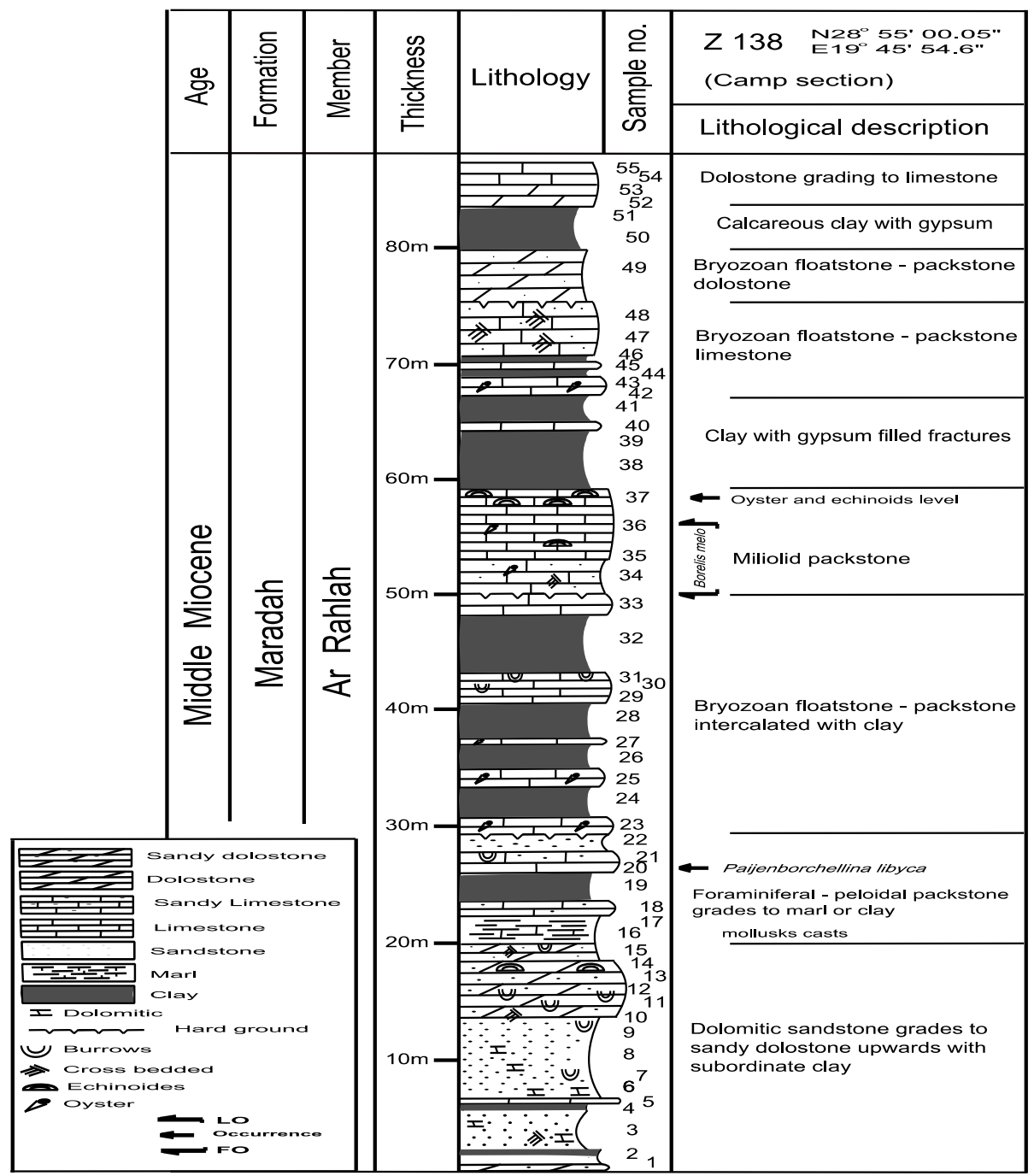

FAUNAL ASSOCIATION

Among the other microfossils common group in the studied mateials are foraminifera and ostracods

\section{Foraminifera}

The microfossils retrieved from Ar Rahlah Member of Maradah Formation at Z138 in Jabal Zaltan area are represented by Foraminifera, Ostracoda and Bryozoa, (All samples were processed for calcareous nannofossils and found to be completely barren. In some horizons, however, foraminifers and/or ostracodes may exist, poorly preserved and of very low diversity. Bryozoans (the scope of this paper) on the other hand are found to be abundant, and moderately divers. The foraminifera are represented by sparsely benthic representatives. These include Elphidium macellum, E. minutum, Ammonia beccarii, Lobatula lobatulus, Discorbis cf. perlexus, Tritaxia tricarinata, Borelis melo, Pyrgo sp., Quinqueloculina spp., Bolivina cf. fastigia, Amphistegina sp., Pararotalia serrata and Rotalia viennesis. Planktonic foraminifers are rare, including Globorotalia cf. opima and Globigerina praebulloides. The distribution of these foraminifers is vertically discontinuous, interrupted by non-fossiliferous clays or sandy dolostone (Fig. 4). 


\section{Ostracods}

Ostracods on the other hand are mainly represented by common Paijenborchellina libyca associated with very rare of Loculicytheretta sp. which are retrieved from the lower part of the studied Z138 (Fig. 4). The presence of Paijenborchellina libyca in the lower part of the section is an indicative of shallow warm marginal marine.

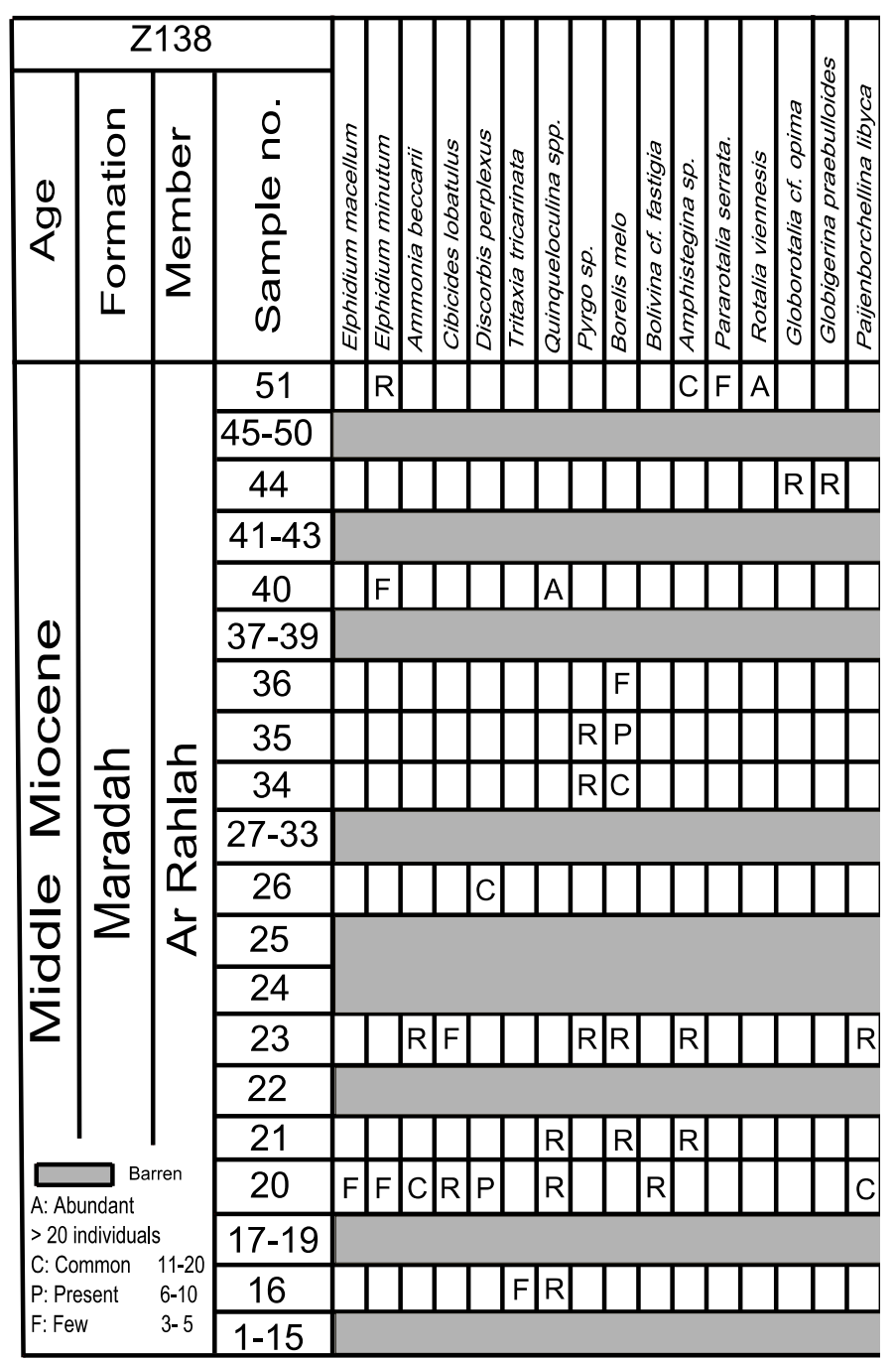

Fig. 4: Foraminifers and ostracodes distribution chart of Ar Rahlah Member of Marada Formation at P138, Jabal Zaltan

\section{Bryozoa from Maradah Formation}

The measured section Z138 at Jabal Zaltan (Figs. 1,2) was studied in detail using Bryozoan assemblages, as this macrofossils group is predominant at several horizons (Fig. 3). The only attempts to document the bryozoans in Maradah Formation have been conducted by El-Hawat (1975). He identified Idmonea spp., Haloporella sp. and Trigonopora moniliferum in petrographic thin sections from the exposed rocks at Zabal Zaltan area.

\section{Z138 Bryozoan analysis}

Out of 32 studied bryozoan species from Maradah Formation (Fig. 5), 15 are encrusting (48\%) and the rest 16 are erecting. The encrusting species are represented by Membraniporiforms and Celleporifors. The erect bryozoans $(52 \%)$ are represented by erect rigid growth form $(56 \%)$, and erect flexible growth form (44\%). In the study area, oyster shells provide the most attachments for the encrusting bryozoans. The limestones and marls contained erect forms, which indicate inter-subtidal (10-50m) environments characterized by moderate rate of sedimentation and relatively strong wave actions. 
Miocene bryozoans from Jabal Zaltan, Northeast Sirt basin

\begin{tabular}{|c|c|c|c|c|c|c|c|c|c|c|c|c|c|c|c|c|c|c|c|c|c|c|c|c|c|c|c|c|c|}
\hline$\stackrel{80}{<}$ & 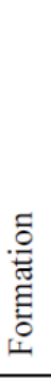 & 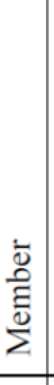 & 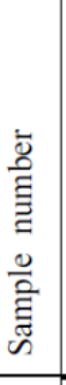 & 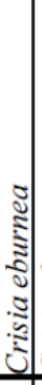 & 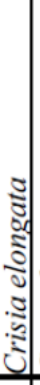 & 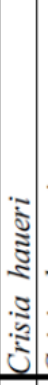 & 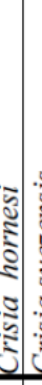 & 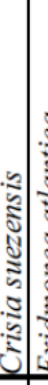 & : & & 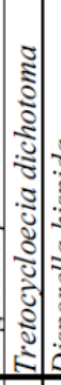 & 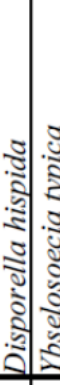 & 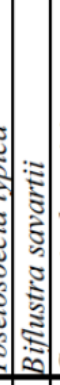 & 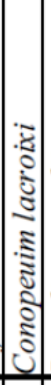 & 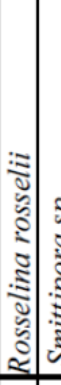 & 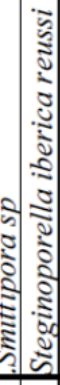 & . & 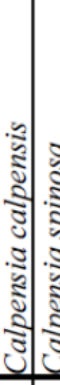 & 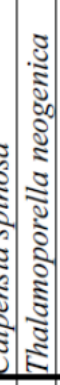 & 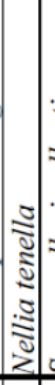 & 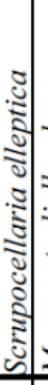 & 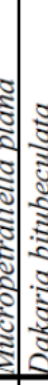 & 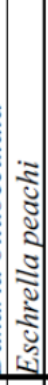 & 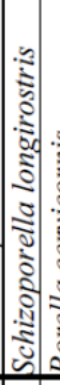 & : & 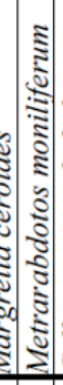 & 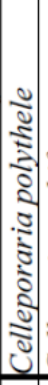 & 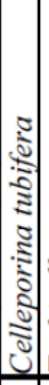 & \\
\hline \multirow{18}{*}{ 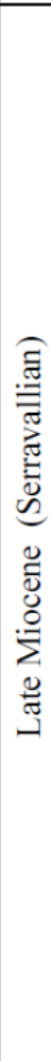 } & \multirow{18}{*}{ 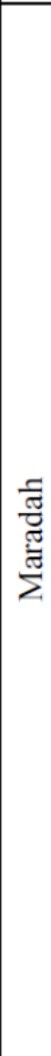 } & & 51 & $\mathrm{C}$ & C & & & & & & & & & & & & & & & \begin{tabular}{|l|l|}
$F$ & \\
\end{tabular} & & & & & & $\mathrm{R}$ & & C & \\
\hline & & & 50 & & & & & & & & & & & & & & & & & & & & & & & $\mathrm{R}$ & & $F$ & \\
\hline & & & 49 & $\mathrm{C}$ & $\mathrm{F}$ & C. & $\mathrm{A} C \mathrm{C}$ & $\mathrm{C}$ & & & & & & & & & & & & C & & & C & & C & $\mathrm{F}$ & C & $F$ & $\mathbf{N}$ \\
\hline & & & 47 & & & & & $\mathrm{C}$ & $c$ & & & & & & & & & & & & & & & & C & $\mathrm{F}$ & C & & \\
\hline & & & 46 & & & & & & & & & & & & & & & & & \begin{tabular}{l|l}
$C$ & 1
\end{tabular} & $\mathrm{R}$ & & & & & $\mathrm{R}$ & & & \\
\hline & & & 43 & $\mathrm{R}$ & C & C & $\mathrm{C}$ & C & C & & & \begin{tabular}{l|l}
$\mathrm{R}$ & $\mathrm{R}$ \\
\end{tabular} & & & & & & & & & C & $\begin{array}{lll}C & R \\
C\end{array}$ & $\mathrm{R}$ & & $\mathrm{R}$ & $\mathrm{R}$ & & & \\
\hline & & & 40 & $\mathrm{R}$ & $\mathrm{R}$ & & $\mathrm{R}$ & & & & & & & & & & & & & \begin{tabular}{|l|l}
$F$ & \\
\end{tabular} & $\mathrm{C}$ & & & & $R$ & $\mathrm{C}$ & & & \\
\hline & & & 35 & & & & & & & & & & & & & & & & & & & & $\mathrm{R}$ & & & & & & \\
\hline & & $\frac{\pi}{\pi}$ & 31 & & $\mathrm{R}$ & & & & & & & & & & & & & & & $\mathrm{F}$ & $\mathrm{R}$ & & & & $\mathrm{F}$ & & & & \\
\hline & & $\stackrel{\square}{\square}$ & 29 & & $\mathrm{R}$ & & & & F & & & & & & & & & & & \begin{tabular}{l|l}
$\mathrm{R}$ & $\mathrm{C}$ \\
\end{tabular} & C & C & & $\mathrm{C}$ & C & $\mathrm{R}$ & & & \\
\hline & & & 27 & $\mathrm{R}$ & $\mathrm{R}$ & & & $\begin{array}{ll}\mathrm{R} & \mathrm{F}\end{array}$ & $R$ & & & & & & & & & & & \begin{tabular}{l|l}
$\mathrm{R}$ & 1 \\
\end{tabular} & $\begin{array}{lll}R & 1 \\
\end{array}$ & $\mathrm{R}$ & & $\mathrm{R}$ & & $\mathrm{F}$ & & & \\
\hline & & & 26 & & & & & & & & & & & & & & & & & C & C & & & & & & & & \\
\hline & & & 25 & & & & & & & & & & $\mathrm{R}$ & $\mathrm{R}$ & \begin{tabular}{l|l}
$\mathrm{R}$ & $\mathrm{R}$
\end{tabular} & $\begin{array}{lll}\mathrm{R} & \mathrm{R}\end{array}$ & $\mathrm{R}$ & $\mathrm{R}$ & & & & & & & C & $F$ & & $\mathrm{R}$ & \\
\hline & & & 23 & & $\mathrm{R}$ & & $\begin{array}{lll}\mathrm{R} & \mathrm{I}\end{array}$ & $\mathrm{R} / \mathrm{F}$ & \begin{tabular}{l|l}
$\mathrm{R}$ & $\mathrm{R}$
\end{tabular} & $\mathrm{R}$ & $\mathrm{R}$ & & & & C & & & $\mathrm{R}$ & $\mathrm{R}$ & C & $\mathrm{C}$ & \begin{tabular}{l|l}
$\mathrm{R}$ & $\mathrm{R}$ \\
\end{tabular} & $\mathrm{R}$ & $\mathrm{R}$ & & & $\mathrm{F}$ & $\mathrm{R}$ & $\mathrm{R}$ \\
\hline & & & 21 & & & & & & $\mathrm{R}$ & & & & & & & & & & & \begin{tabular}{l|l}
$\mathrm{R}$ & $\mathrm{I}$
\end{tabular} & $\mathrm{R}$ & & & & & & $\mathrm{R}$ & & \\
\hline & & & 20 & $\mathrm{R}$ & $\mathrm{R}$ & & C & & $R$ & & & & & & & & & & & \begin{tabular}{l|l}
$\mathrm{R}$ & $\mathrm{I}$
\end{tabular} & $\mathrm{R}$ & . & & & & & $\mathrm{R}$ & & \\
\hline & & & 17 & & & & & & & & & & & & & & & & & & & & & & & $\mathrm{R}$ & & & \\
\hline & & & 16 & & & & & & & & & & & & & & & & & $\begin{array}{lll}\mathrm{R} & \mathrm{I}\end{array}$ & $\mathrm{R}$ & & & & & & $\mathrm{F}$ & & \\
\hline
\end{tabular}

Fig. 5: Bryozoan distribution chart of Ar Rahlah Member, Maradah Formation at Z138 section in Jabal Zaltan area. (R: Rare 1-2; C: Common 3-5; F: Frequent 6-10; A: Abundant 10)

\section{Systematic Paleontology}

Thirty-two bryozoan species are taxonomic studied of all recorded species (Fig. 5). The study is based primarily on the classification of Bassler (1953) with modifications. In addition to the morphologic description of two new species, micrometric measurements, distribution, and habitat for each species are given. The illustrated types are deposited in the Department of Earth Sciences, Faculty of Science, University of Benghazi. The used parameters, statistics and abbreviations, as well as the form in which are presented, are as follows:

\begin{tabular}{|ccccc|}
\hline Parameter & $\begin{array}{c}\text { No. of measured } \\
\text { Zoaria, Zooecia }\end{array}$ & Range & Standard deviation & Mean \\
\hline $\mathrm{X}^{*}$ & $(4,8)$ & $0.125-0.301 \mathrm{~mm}$ & $(0.021)$ & $0.332 \mathrm{~mm}$ \\
\hline
\end{tabular}

$\mathrm{Dz}=$ Zoarial diameter; $\mathrm{Dt}=$ Diameter of the zooecial tubes; $\mathrm{Dp}=$ Peristomes diameter; $\mathrm{Do}=$ Orifice diameter; $\mathrm{Df}=$ Fascicles diameter; $\mathrm{Lz}=$ Zooecial length; $\mathrm{l}=$ Zooecial width; $\mathrm{Lo}=$ Apertural or Opesial length; lo = Apertural or Opesial width; Lov $=$ Ovicell length; Iov $=$ Ovicell width; Lav $=$ Avicularian length; Iav $=$ Avicularian width. 
El-Safori, Y. A. and Muftah, A. M.

Class Stenolaemata Borg, 1926

Order: Cyclostomata Busk, 1852

Family: Crisidae Johnston, 1847

Crisia eburnea (Linnaeus, 1758)

(Fig. 6/ 1)

1758 Sertularia eburnea Linnaeus: 810.

1982 Crisia. eburnea Winston, p. 155, fig. 91.

Measurements: Dz (3) 0.2646-0.266 (0.083) 0.259mm; Lz (1, 3) 0.351-0.389 (0.083) 0.365mm; Do (1, 10) $0.064-0.070$ (0.024) $0.068 \mathrm{~mm} ; \mathrm{Dp}(1,10) 0.079-0.089(0.102) 0.087 \mathrm{~mm}$.

Material: Maradah Formation, Z138, Sample nos. 20, 27, 40, 43, 49, 51.

Distribution: Common in the cold waters of Europe and America, also in western Atlantic, Mediterranean Sea and West Africa.

Habitat: It is always dominant at $50 \mathrm{~m}$, with a maximum depth of $300 \mathrm{~m}$ (Hayward and Ryland, 1985).

Remarks: The present species has a close relation to Crisia denticulata, but differs in having only smaller size ranges and not-wedged basis rami.

\section{Crisia elongata Milne-Edwards, 1838}

1838 Crisia elongata Milne-Edwards, p. 203, pl. 7, fig. 2.

1995 Crisia elongata Ziko and El-Sorogy, p. 82, figs. 3: 1-2.

Measurements: Dz (5) 0.215-0.270 (0.028) 0.260mm; Lz (1, 10) 0.410-0.473 (0.019) 0.455mm; Do (2, 10) $0.064-0.070$ (0.022) $0.068 \mathrm{~mm}$; Dp $(2,10)$ 0.076-0.086 (0.015) $0.083 \mathrm{~mm}$; Lov $(2,5)$ 0.491-0.500 (0.022) 0.495mm; Iov $(2,5)$ 0.298-0.313 (0.012) $0.305 \mathrm{~mm}$.

Material: Maradah Formation, Z138, Sample nos. 20, 23, 27, 29, 31, 40, 43, 49, 51.

Distribution: Eocene (France, and North America); Oligocene (France, Germany, and Italy); Miocene (Egypt, CSSR, France, Hungary, Italy, and Austria); Pliocene (Italy), Pleistocene (Egypt).

Habitat: Atlantic, Mediterranean, Red Sea, Japan, with a depth range of 0-59m (Vavra, 1977).

Crisia hauri (Reuss, 1848)

(Fig. 6/2)

1848 Crisia hauri Reuss, p. 54, pl. 7, figs. 22-24.

2002 Crisia hauri El Safori, p. 426, pl. 2, fig. 2.

Measurements: Dz (5) 0.184-0.200 (0.023) 0.192mm; Lz (1, 10) 0.152-0.167 (0.017) 0.166mm; Do (2, 10) 0.060-0.063 (0.062) 0.062mm; Dp (2, 10) 0.086-0.094 (0.075) 0.090mm.

Material: Maradah Formation, Z138, Sample nos. 43, 49.

Distribution: Eocene (Egypt, and Italy); Oligocene (Germany); Miocene (Egypt, France, Italy, Austria, Morocco) .

Habitat: Common in the cold waters of Europe and America, also in western Atlantic, Mediterranean Sea and West Africa.

Crisia hornesi Reuss, 1848

(Fig. 6/3)

1848 Crisia hornesi Reuss, p. 54, pl. 7, fig. 21. 
Miocene bryozoans from Jabal Zaltan, Northeast Sirt basin

2002 Crisia hornesi El Safori, p. 426, pl. 2, fig. 3.

Measurements: Dz (3) 0.489-0.522 (0.023) 0.510mm; Lz ((1, 3) 0.351-0.389 (0.013) 0.365mm; Do (2, 10) $0.064-0.070(0.004) 0.068 \mathrm{~mm} ; \mathrm{Dp}(2,10) 0.076-0.086(0.009) 0.081 \mathrm{~mm}$.

Material: Maradah Formation, Z138, Sample nos. 20, 23, 40, 43, 49.

Distribution: Eocene (France, Italy, and North America); Oligocene (Germany, France, Italy, and USA); Miocene (Egypt, CSSR, Greece, Italy, Poland, Romania, Hungary, and Portugal); Pliocene-Pleistocene of Italy.

Habitat: Red Sea, Philippines, at depth from 100 to $300 \mathrm{~m}$, temperature: $11.2^{\circ} \mathrm{C}$.

Crisia suezensis El Safori, 1994

(Fig. 6/4)

1994 Crisia suezensis El Safori, p. 109, pl. 9, figs. 7, 8.

2010 Crisia suezensis Ziko et al., p. 67, pl. 1, figs. 8-9.

Measurements: Dz (3) 0.520-0.669 (0.023) 0.605mm; Lz (1, 10) 0.232-0.320 (0.022) 0.284mm; Do (2, 10) 0.058-0.086 (0.014) 0.073mm; Dp $(2,10) 0.083-0.100(0.012) 0.095 \mathrm{~mm}$.

Material: Maradah Formation, Z138, Sample nos. 23, 27, 43, 47, 49.

Distribution: Miocene of Egypt.

Family: Tubuliboridae Johnston, 1838

Exidmonea atlantica (Forbes in Johnston, 1847)

(Fig. 6/5)

1847 Idomnea atlantica Forbes in Johnston, 278, pl. 48, fig. 3.

2002 Exidmonea atlantica El Safori, p. 427, pl. 2, fig. 7.

Measurements: Dz (5) 0.641-0.769 (0.023) 0.727mm; Dp $(2,10) 0.111-0.134$ (0.014) 0.126mm; Df (2, 6) $0.387-0.410(0.010) 0.390 \mathrm{~mm}$.

Material: Maradah Formation, Z138, Sample nos. 23, 27, 47.

Distribution: Eocene (Italy, Hungary, Romania, USA? and Argentina?); Oligocene (Germany, Italy, and USA?); Miocene (Egypt, France, Italy, Austria, Poland, and Morocco); Pliocene (Tunisia, Morocco, Mexico?); Pleistocene (Italy, Canada?).

Habitat: Cosmopolitan, variable depth $(0-850 \mathrm{~m})$, with an optimum depth range of $40-100 \mathrm{~m}$ in the Mediterranean Sea (Moissette, 1988).

Exidmonea undata (Reuss, 1851)

(Fig. 6/6)

1851 Idmonea undata Reuss, p. 172, p1. 9, fig. 20

2010 Exidmonea undata Zagoršek, p. 29, pl. 7, figs. 1-4.

Measurements: Dz (5) 0.435-0.476 (0.024) 0.456mm; Dp (1, 10) 0.110-0.129 (0.014) 0.117mm; Df (1, 6) $0.354-0.362(0.010) 0.358 \mathrm{~mm}$.

Material: Maradah Formation, Z138, Sample no. 23 (Fig. 5).

Distribution: Eocene (Italy, Hungary, Romania, USA?, and Argentina?); Oligocene (Germany, Italy, and USA?); Miocene (Egypt, France, Italy, Austria, Poland, and Morocco); Pliocene (Tunisia, Morocco, Mexico?); Pleistocene (Italy, Canada?). 
El-Safori, Y. A. and Muftah, A. M.

Habitat: Cosmopolitan, variable depth $(0-850 \mathrm{~m})$, with an optimum depth range of $40-100 \mathrm{~m}$ in the Mediterranean Sea (Moissette, 1988).

Family: Cerioporidae Reuss, 1866

Tretocycloecia dichotoma Canu, 1919

1848 Heteropora dichotoma Reuss, p. 35, pl. 5, fig. 20.

2010 Tretocycloecia dichotoma Ziko, et al., p. 92, pl. 4, fig. 12, pl. 5, fig. 1.

Measurements: Dz (2) 1.300-1.510 (0.083) 1.387mm; Do (1, 10) 0.064-0.085 (0.024) 0.076mm; Dp (1, 10) $0.088-0.129(0.108) 0.116 \mathrm{~mm}$.

Material: Maradah Formation, Z138, Sample no. 23.

Distribution: Miocene (Egypt, Austria); Tertiary (France).

Family: Lichenoporidae Smitt, 1867

Disporella hispida (Fleming, 1828)

(Fig. 6/7)

1828 Discopora hispida Fleming, p. 530.

2010 Disporella cf. hispida Zágoršek, p.39, pl. 36, figs. 2-5.

Measurements: Dz (5) $0.464-0.640$ (0.043) 0.587mm; Do (1, 10) 0.075-0.108 (0.054) 0.098mm; Dp (1, 10) 0.133-0.144 (0.054) 0.136mm; Df (1, 6) 0.197-0.209 (0.075) $0.201 \mathrm{~mm}$.

Material: Maradah Formation, Z138, Sample no. 43.

Distribution: Eocene (Egypt); Oligocene (Italy, Germany); Miocene (Egypt, France, Italy, Austria, Hungary, and CSSR); Pliocene (England and Italy); Pleistocene (USA and Italy). It inhabits the Mediterranean Sea, Arctic, Atlantic and Pacific Oceans (Vavra, 1974).

Family Diaperoeciidae Canu, 1918

Ybselosoecia typica (Manzoni, 1878)

(Fig. 6/8)

1878 Filisparsa typica Manzoni p. 10, pl. 8, fig. 30.

2010 Ybselosoecia typica Zagoršek p. 31,pl. 15, fig. 1-5.

Measurements: Dz (2) 1.250-1.610 (0.023) 1.330mm; Lz (2, 10) 0.456-0.487 (0.012) 0.466mm; Do (2, 10) $0.133-0.1455$ (0.014) $0.143 \mathrm{~mm}$; $\mathrm{Dp}(2,10) 0.157-0.188(0.012) 0.170 \mathrm{~mm} .$.

Material: Maradah Formation, Z138, Sample no. 43.

Distribution: Eocene Europe (Poland, France, Romania); Oligocene (USA); Miocene Austria, Poland, France, Italy); Quaternary and Recent (Italy).

Order Cheilostomata Busk, 1852

Suborder Malacostegina Levinsen, 1909

Family: Membraniporidae Busk, 1852

Biflustra savartii (Audouin, 1826)

1826 Flustra savartii Audouin: 240, pl. 10, fig. 10.

1988 Biflustra savartii Moissette: 73, pl. 11, figs. 6, 9. 
Miocene bryozoans from Jabal Zaltan, Northeast Sirt basin

Measurements: Lz $(2,10) 0.468-0.546(0.124) 0.513 \mathrm{~mm}$; Iz $(2,10) 0.260-0.312$ (0.125) $0.297 \mathrm{~mm}$; Lo $(1,10) 0.234-0.312$ (0.143) 0.260mm; Io $(2,10) 0.208-0.260(0.137) 0.223 \mathrm{~mm}$.

Material: Maradah Formation, Z138, Sample nos. 23, 25.

Distribution: Eocene (France, Spain, Egypt, Italy, and USA); Oligocene (Germany, USA and Italy); Miocene (France, Egypt, Austria, Czechoslovakia, Poland, Italy, Tunisia, Libya, USA, and Portugal); Pliocene (England, Spain, Netherlands, Portugal, Italy, Tunisia, Venezuela, and Australia); Pleistocene (Italy, Algeria, USA, china, and Argentina).

Habitat: Cosmopolitan in Atlantic, Indian and Pacific Oceans. It survives in Equatorial hot waters to a depth of 100m (Moissette, 1988).

Family Electridae d'Orbigny, 1851

Conopeuim lacroixii (Audouin, 1826)

(Fig. 7/ 1)

1826 Flustra lacroixi Audouin: 240, pl. 10, fig. 9.

1912 Membranipora lacroixi Canu: 195, pl. 10, fig. 5, 6.

Measurements: Lz $(2,10) 0.475-0.550$ (0.024) $0.515 \mathrm{~mm} ; \operatorname{Iz}(2,10) 0.275-0.375(0.020) 0.340 \mathrm{~mm} ; \mathrm{Lo}(2$, 10) $0.225-0.256$ (0.023) $0.238 \mathrm{~mm}$; Io $(2,10) 0.199-0.249(0.014) 0.218 \mathrm{~mm}$.

Occurrence: Maradah Formation, Z138, Sample no. 25 (Fig. 5).

Distribution: Eocene (France, Belgium, Egypt and USA); Miocene (Egypt, USA Tunisia, Italy, Austria, and Hungary); Pliocene (England); Quaternary (England, and Argentina).

Habitat: Atlantic, Pacific and Indian Oceans, Mediterranean and Red Sea. Shore depths (Canu and Bassler, 1920).

Family Antroporidae Vigneaux, 1949

Rosselina rosselii (Audouin, 1826)

1826 Flustra rosselii Audouin, p. 240, pl. 10, fig.1.1.

2002 Rosselina rosselii El Safori, p. 437, pl. 4, fig. 6.

Measurements: Lz $(2,10) 0.375-0.427$ (0.074) 0.394mm; Iz (2, 10) 0.275-0.303 (0.060) 0.289mm; Lo (2, 10) $0.100-0.124$ (0.053) $0.109 \mathrm{~mm}$; Io $(2,10) 0.100-0.126(0.050) 0.113 \mathrm{~mm}$; Lav $(2,3) 0.127-0.153$ (0.063) 0.138mm, Iav $(2,3) 0.075-0.087(0.020) 0.081 \mathrm{~mm}$.

Material: Maradah Formation, Z138, Sample no. 25.

Distribution: Miocene (Egypt, France, Spain, and Portugal); Pliocene (Tunisia); Pleistocene (Italy).

Family Onychocellidae Jullien, 1882

Smittipora sp.

(Fig. 7/2)

Figured material: Maradah Formation, Z138, Sample no. 25.

Description: Zooecia encrusting unilamellar, hexagonal with mainly regular alternating rows of zoeecia, separated by fine furrows. Cryptocyst is finely granulated, slightly convex. Opesia is large oval to elliptical. Fertile zooecia have the same size with larger opesia and rounded anter. Avicularian zooecia with wide and salient proximal part, and straight narrow rostrum.

Measurements: Lz $(2,10)$ 0.450-0.540 (0.024) 0.544mm; Iz (2, 10) 0.295-0.340 (0.020) 0.320mm; Lo (2, 10) $0.140-0.194$ (0.013) $0.169 \mathrm{~mm}$; Io $(2,10) 0.140-0.176(0.015) 0.155 \mathrm{~mm}$; Lav $(2,3) 0.487-0.520$ (0.025) 0.508mm; Iav $(2,3) 0.335-0.348(0.010) 0.340 \mathrm{~mm}$. 
El-Safori, Y. A. and Muftah, A. M.

Family: Steginoporellidae Bassler, 1953

Steginoporella iberica reussi Pouyet and David, 1979.

1979 Steginaporella iberica reussi Poyet and David, 780, pl. 4, fig. 3, text fig. 3.

Measurements: Lz $(1,10)$ 0.985-1.009 (0.017) 0.998mm; Iz (1, 10) 0.655-0.610 (0.008) 0.600mm; Lo (1, 10) 0. 124-0.256 (0.022) $0.245 \mathrm{~mm}$; Io $(1,10) 0.379-0.400(0.010) 0.386 \mathrm{~mm}$

Material: Maradah Formation, Z138, Sample no. 25.

Distribution: Miocene (Vienna Basin-Austria, Rhone Basin-France).

Family: Calpenciidae Canu and Bassler, 1923

Calpensia calpensis (Busk, 1854)

1854 Membranipora calpensis Busk: 60 pl. 104, figs. 5-6.

2002 Calpensia calpensis El Safori, p. 437, pl. 5, fig. 1.

Measurements: Lz $(1,10)$ 0.598-0.702 (0.064) $0.657 \mathrm{~mm}$; Iz $(1,10)$ 0.390-0.464 (0.085) 0.438mm; Lo (1, 10) $0.090-0.110(0.040) 0.104 \mathrm{~mm}$; Io $(1,10) 0.130-0.200(0.030) 0.148 \mathrm{~mm}$.

Material: Maradah Formation, Z138, Sample no. 25.

Distribution: Miocene (Egypt, France, and Portugal); Pliocene (Italy, Tunisia); Pleistocene (Italy); Quaternary (Italy). It is of Mediterranean range (David et al., 1972).

Calpensia gracilis (Münster in Goldfuss, 1826)

(Fig. 7/3)

1847 Membranipora gracilis Münster, p. 93, pl. 11, fig. 12

2010 Calpensia gracilis Zagoršek, p. 47, pl. 3, figs. 1-4.

Measurements: Lz $(1,10) 0.598-0.702(0.024) 0.657 \mathrm{~mm}$; Iz $(1,10)$ 0.390-0.464 (0.015) 0.438mm; Lo $(1,10) 0.090-0.110$ (0.010) 0.104mm; Io $(1,10) 0.130-0.200(0.010) 0.148 \mathrm{~mm}$.

Material: Maradah Formation, Z138, Sample no. 25.

Distribution: Miocene (France, Austria, Morocco, Algeria, Poland, and Romania).

\section{Calpensia spinosa $\mathrm{n}$. sp.}

(Fig. 7/ 4)

Material: Holotype, Maradah Formation, Ar Rahlah Member, Z138 section Serravallian, Z138, Sample no. 23 .

Etymology: The species is named after the specific criteria of mural spine occurrences.

Diagnosis: Calpensia having zooecia with one or two spines at proximal cadre.

Description: Zoarium encrusts membraniporiform. Zooecia distinct, lozenge, arranged in alternating longitudinal rows, separated by thin furrows. Mural rim thin, convex, slightly salient, granulated, basal part isoften pierced by two and rarely one small spine with thick base and abraded shaft. Cryptocyst shallow, slightly convex to flat, finely granulated and perforated, grooved by two small symmetrical rounded opesiuels placed just below the proximal border of opesia. Opesia elliptical with rounded distal and concave to slightly concave proximal border; peristome thin, salient. Ovicells are not observed.

Measurements: Lz $(1,10)$ 0.423-0.452 (0.014) $0.432 \mathrm{~mm}$; Iz (1, 10) 0.273-0.334 (0.021) 0.329mm; Lo (1, 10) 0.062 - 0.072 (0.009) $0.065 \mathrm{~mm}$; Io $(1,10) 0.086-0.097$ (0.010) $0.093 \mathrm{~mm}$.

Affinity: This species is close to C. calpensis, but its mural rim is often pierced by two and rarely one small spine. 
Miocene bryozoans from Jabal Zaltan, Northeast Sirt basin

Family Thalamoporellidae Levinsen, 1902

Thalamoporella zaltaniensis $\mathrm{n}$. sp.

(Fig. 7/ 5, 6)

Material: Holotype, Maradah Formation, Serravallian, Ar Rahlah Member, Z138 section, Sample no.

Etymology: The species is named after Jabal Zaltan.

Diagnosis: Thalamoporella having flat cryptosyst with two mediun opesiules situated at the middle part of the cryptocyst.

Description: Zoarium encrusts and membraniporiform. Zooecia distinct, arranged in alternating longitudinal rows and separated by thin furrows. Mural rim thin, convex, slightly salient, granulated, basal part pierced by two and rarely one small spines with thick base and abraded shaft. Cryptocyst shallow, little convex to flat, finely granulated and perforated, grooved by two small symmetrical rounded opesiuels, placed just below the proximal border of the opesia. Opesia elliptical with rounded distal and concave to little concave proximal border; peristome thin, salient. Ovicells are not observed.

Measurements: Lz (2, 7) 0.391-0.492 (0.036) 0.449mm; Iz (2, 7) 0.187-0.282 (0.042) 0.253mm; Lo (2, 7) $0.043-0.057(0.013) 0.050 \mathrm{~mm}$; Io $(2,7) 0.101-0.108(0.006) 0.104 \mathrm{~mm}$; Lav $(1,2) 0.558-0.565$ (0.013) $0.561 \mathrm{~mm} ; \operatorname{Iav}(1,2) 0.276-0.284(0.006) 0.280 \mathrm{~mm}$.

Affinity: This species is close to Th. neogenica but it has lager opesules, more flattened cryptocyst, opesia with typical elliptical shape.

Family Scrupocellariidae Levinsen, 1909

Nellia tenella (Lamarck, 1816)

(Fig. 7/ 7)

1816 Cellaria tenella Lamarck: 135.

1974 Nellia tenella David and Pouyet, p. 132.

Measurements: Lz $(2,10) 0.373-0.452(0.014) 0.397 \mathrm{~mm} ; \quad$ Iz $(2,10) 0.123-0.164$ (0.010) $0.252 \mathrm{~mm}$; Lo $(2,10) 0.242-0.262$ (0.013) 0.254mm; Io $(2,10) 0.086-0.117(0.010) 0.103 \mathrm{~mm}$.

Material: Maradah Formation, Z138, Sample nos. 16, 20, 21, 23, 26, 27, 29, 31, 40, 46, 49, 51.

Distribution: Eocene (France); Eocene-Oligocene (USA); Miocene (Egypt, Jamaica, Austria); PliocenePleistocene (USA).

Family Scrupocellariidae Levinsen, 1909

Scrupocellaria elleptica (Reuss, 1848)

1848 Bactridium elleptica Reuss, p. 148, pl. 11, figs. 1-9.

1996 Scrupocellaria elleptica Haddadi-Hamdane, p. 73, pl. 5, fig. 5.

Measurements: Lz $(2,5)$ 0.368-0.437 (0.035) $0.410 \mathrm{~mm}$; Iz $(2,5)$ 0.138-0.184 (0.016) 0.176mm; Lo $(2,5)$ 0.189-0.253 (0.018) 0.228mm; Io $(2,5)$ 0.069-0.119 (0.010) $0.103 \mathrm{~mm}$.

Material: Maradah Formation, Z138, Sample nos. 16, 20, 21, 23, 26, 27, 29, 31, 40, 46.

Distribution: Eocene (France, Italy, Hungary, and France); Oligocene (Italy and France); Miocene (Egypt, Libya, France, Iran, Portugal, Austria, and Belgium); Pliocene (Portugal, Spain, Italy, and Tunisia); Pleistocene (Egypt, Algeria, and Italy).

Infraorder: Ascophorina Levinsen, 1909

Family: Mucronellidae Levinsen, 1902

Mucropetraliella plana Moissette, 1988 


\section{El-Safori, Y. A. and Muftah, A. M.}

(Fig. 8/ 1)

1988 Mucropetraliella plana Moissette, p. 121, pl 20, figs. 1-3.

Measurements: Lz $(4,10) 0.840-0.920(0.024) 0.820 \mathrm{~mm}, \mathrm{Iz}(4,10) 0.420-0.570(0.030) 0.440 \mathrm{~mm}$, Lo $(4,15)$ 0.201- $0.224(0.023) 0.214 \mathrm{~mm}$, Io $(4,10) 0.223-0.265$ (0.016) $0.240 \mathrm{~mm}$, Lav $(4,7) 0.103-0.113$ (0.006) 0.108mm, Iav $(4,7) 0.007-0.008(0.006) 0.007 \mathrm{~mm}$,

Material: Maradah Formation, Z138, Sample nos. 20, 23, 27, 43 (Fig. 5).

Distribution: Miocene (Egypt, Italy, Germany, Austria, France); Pliocene (Italy, England, Belgium, PaysBas); Pleistocene (England); Quaternary (Italy).

Watersiporidae Vigneaux, 1949

Watersipora bitubeculata (Canu and lecointre, 1928)

(Fig. 8/2)

1928 Dakaria bitubeculata Canu and lecointre, p. 68, pl. 12, figs. 3-6.

1988 Dakaria cf. bitubeculata Moissette, p. 129, pl. 21, figs. 1-2.

Measurements: Lz $(2,10) 0.561-0.625$ (0.042) $0.590 \mathrm{~mm}$; Iz $(2,10) 0.283-0.323(0.040) 0.308 \mathrm{~mm}$; Lo $(2,10) 0.154-0.160$ (0.013) $0.158 \mathrm{~mm}$; Io $(2,10) 0.152-0.162(0.012) 0.156 \mathrm{~mm}$.

Material: Maradah Formation, Z138, Sample nos. 23, 29, 43.

Distribution: Miocene (France and Algeria).

$$
\begin{gathered}
\text { Romancheinidae Jullien, } 1888 \\
\text { Eschrella peachi (Johnston, 1847) }
\end{gathered}
$$

1847 Eschrella peachi Johnston, 315, pl. 55, fig. 5.

1988 Eschrella peachi Moissette, p. 155, pl. 24, fig 122, pl. 13, fig. 6.

Measurements: Lz $(1,10) 0.325-0.346(0.014) 0.338 \mathrm{~mm} ; \quad$ Iz $(1,10) 0.283-0.323$ (0.024) $0.308 \mathrm{~mm}$; Lo $(1,10)$ 0.081-0.094 (0.0093) 0.092mm; Io $(1,10)$ 0.090- 0.096 (0.004) 0.092mm; Lav $(1,10) 0.161-$ 0.173 (0.007) 0.171mm; Iav $(1,10) 0.173-0.232(0.014) 0.210 \mathrm{~mm}$.

Material: Maradah Formation, Z138, Sample nos. 23, 35, 43, 49 (Fig. 5).

Distribution: Mediterranean, Arctic and East Atlantic range. Miocene (France); Pliocene (Italy, England); Pleistocene (England); Pleistocene (Italy).

Schizoporellidae Jullien, 1883

Schizoporella longirostris (Canu and Bassler, 1930)

1930 Schizoporella longirostris Canu and Bassler, 43, pl.4, figs.15-20, pl.5, figs.1-19.

1989 Schizoporella longirostris Schmidt, p. 39, pl. 11, figs. 5-8.

Measurements: Lz $(2,10) 0.393-0.452(0.074) 0.422 \mathrm{~mm} ; \quad$ Iz $(2,10) 0.232-0.374$ (0.060) 0.353mm; Lo $(2,10) 0.082-0.094$ (0.053) 0.089mm; Io $(2,10) 0.012-0.014$ (0.050) $0.013 \mathrm{~mm}$; Lav $(2,10) 0.135-0.193$ (0.074) $0.182 \mathrm{~mm}$; Iav $(2,10) 0.060-0.130(0.060) 0.094 \mathrm{~mm}$.

Material: Maradah Formation, Z138, Sample nos. 23, 27, 29 (Fig. 5).

Distribution: Miocene (Italy, Rhone Basin-France, Algeria); Pliocene (Italy); Pleistocene (Italy).

Habitat: East Atlantic, Mediterranean, Red Sea. The present species range from 0.5 to $150 \mathrm{~m}$ with 20 60m optimum range, Canu and Bassler (1930). 
Miocene bryozoans from Jabal Zaltan, Northeast Sirt basin

Bryocryptellidae Vigneaux, 1949

Porella cervicornis (Pallas, 1766)

(Fig. 8/3)

1766 Millepora cervicornis Pallas. p. 252.

1989 Smittina cervicornis Bishop and Hayward, p. 30, figs.. 114-118.

Measurements: Lz $(2,10) 1.750-1.960$ (0.032) $0.795 \mathrm{~mm} ;$ Iz $(2,10) 0.348-0.454$ (0.036) $0.374 \mathrm{~mm}$; Lo $(2,10) 0.164-0.243$ (0.023) 0.193mm; Io $(2,10) 0.147-0.162(0.022) 0.145 \mathrm{~mm}$.

Material: Maradah Formation, Z138, Sample nos. 25, 29, 31.

Distribution: Oligocene (Italy); Miocene (Egypt, Morocco, France, Italy, Portugal, Austria); Pliocene (Bays-Bas, Portugal, Spain, Italy, Tunisia, Algeria); Pleistocene (Italy).

Habitat: Mediterranean, 30-120m depth range, 40-60m optimum depth Atlantic (30-400m depth range); Red Sea (Gautier, 1961).

Family: Margarettidae Harmer, 1957

Margaretta cereoides (Ellis and Solander, 1786)

(Fig. 8/4)

1786 Cellaria cereoides Ellis and Solander, p. 26, pl. 5, figs. B-E.

2002 Margaretta cereoides El Safori, p. 450, pl. 7, fig. 6.

Measurements: Lz (2, 10) 1.430-1.560 (0.030) 1.495mm; Iz (2, 10) 0.468-0.49 (0.026) 0.481mm; Lo $(2,10) 0.130-0.153$ (0.023) 0.147mm; Io $(2,10) 0.172-0.182(0.012) 0.180 \mathrm{~mm}$.

Material: Maradah Formation, Z138, Sample nos. 40, 43, 47, 49 (Fig. 5).

Distribution: Eocene (Spain, Italy, France, and Egypt); Oligocene (Italy, Germany, Austria, Poland, and USA); Miocene (Italy, France, Egypt, Austria, Poland, Romania, Libya, Algeria, and Morocco); Pliocene (Italy, North Africa, and Central America).

Habitat: Adriatic, Mediterranean, Pacific, and Red Sea; Atlantic in tropical and subtropical regions (Schmid, 1989).

Metrarabdotosidae Vigneaux, 1949

Metrarabdotos moniliferum (Milne-Edwards, 1836)

(Fig. 8/5,6)

1836 Eschara monilifera Milne-Edwards, 27, pl. 9, fig. 1.

1989 Metrarabdotos moniliferum Bishop and Hayward, p. 84, figs. 184-185.

Measurements: Lz (4, 6) 0.743-0.792 (0.024) 0.7848mm; Iz (4, 6) 0.283-0.323 (0.020) 0.318mm; Lo (4, 6) $0.132-0.143$ (0.023) $0.134 \mathrm{~mm}$; Io $(4,6) 0.125-0.132(0.013) 0.131 \mathrm{~mm}$; Lav $(4,4) 0.284-0.321(0.015)$ $0.306 \mathrm{~mm}$; Iav $(4,4) 0.132-0.149$ (0.016) $0.142 \mathrm{~mm}$; Lov $(4,3) 0.821-0.943(0.012) 0.886 \mathrm{~mm}$; Iov $(4,3)$ $0.621-0.742(0.028) 0.653 \mathrm{~mm}$.

Material: Maradah Formation, Z138, Sample nos. 17, 25, 27, 29, 40, 43, 46, 47, 49, $50,51$.

Remarks: According to Cheetham, (1968), the M. moniliferum species has non-costulate ovicell. Canu and Bassler (1920), however, mentioned the change of degree of ovicell costulation among the English, French and American specimens, and its non-specific importance.

Distribution: Miocene (Egypt, Italy, Germany, Austria, France); Pliocene (Italy, England, Belgium, Pays-Bas); Pleistocene (England); Quaternary (Italy). 
El-Safori, Y. A. and Muftah, A. M.

Family Lepraliellidae Vigneaux, 1949

Celleporaria polythele (Reuss, 1848)

1848 Cellepora polythele Reuss, p. 77, pl. 9, fig. 18.

2002 Celleporaria polythele E1 Safori, p. 451.

Measurements: Lz $(3,10) 0.325-0.346(0.054) 0.338 \mathrm{~mm} ; \mathrm{Iz}(3,10) 0.283-0.323(0.060) 0.308 \mathrm{~mm}$; Lo $(3,10) 0.081-0.094$ (0.053) 0.092mm; Io $(3,10) 0.090-0.096(0.054) 0.092 \mathrm{~mm}$.

Material: Maradah Formation, Z138, Sample nos. 16, 20, 21, 23, 47, 49.

Remarks: C. polythele is characterized by the absence of advetitous avicularia and the presence of mammelar interzooecial projections.

Distribution: Miocene (Italy, France, Egypt, Austria, Netherlands, CSSR, Hungary).

Family Celleporidae Johnston, 1838

Celleporina tubifera Moissette, 1988

(Fig. 8/ 7, 8)

1988 Celleporina tubifera Moissette, p. 187, pl. 30, figs. 1-3.

Measurements: Lz $(2,10) 0.333-0.427(0.034) 0.370 \mathrm{~mm} ; \mathrm{Iz}(1,10) 0.338-0.386(0.023) 0.372 \mathrm{~mm}$; Lo $(2,10)$ 0.147- $0.174(0.033) 0.163 \mathrm{~mm}$; Io $(1,10) 0.165-0.187$ (0.040) $0.152 \mathrm{~mm}$; Lav $(2,3) 0.359-0.390$ (0.018) $0.378 \mathrm{~mm}$; Iav $(2,3) 0.123-0.143$ (0.026) $0.133 \mathrm{~mm}$.

Material: Maradah Formation, Z138, Sample nos. 23, 25, 49, 50, 51.

Distribution: Miocene (Egypt, and Libya).

Turbicellepora coronopus (Wood, 1844)

(Fig. 8/ 9)

1844 Cellepora coronopus Wood, p. 18.

1992 Turbicellepora coronopus Pouyet and Moissette, p. 79, pl. 12, fig. 7.

Measurements: Lz (1,10) 0.381-0.472 (0.074) 0.445mm; Iz (1, 10) 0.363-0.390 (0.020) 0.382mm;

Lo $(1,10)$ 0.072- 0.109 (0.094) 0.098mm; Io $(1,10) 0.090$ - 0.118 (0.075) $0.109 \mathrm{~mm}$; Lav $(1,10) 0.358$ $0.418(0.093) 0.409 \mathrm{~mm}$. Iav $(1,10) 0.098-0.116(0.029) 0.110 \mathrm{~mm}$.

Material: Maradah Formation, Z138, Sample nos. 16, 23, 49.

Distribution: Miocene (Egypt, and Libya).

\section{PALEOGEOGRAPHIC DISTRIBUTION}

Most of the Neogene bryozoans are preserved in Miocene and Pliocene basins in south Europe (Spain) and North Africa (Tunisia, Algeria, Morocco, Egypt and Libya), with additional occurrences in Germany, France, Austria and Poland, (Fig. 6). This distribution pattern suggests that bryozoans preferred low latitudes in the Mediterranean region. The Plio-Pleistocene bryozoan record, however, is restricted to Tunisia in North Africa and to Spain and Portugal in South Europe. Pouyet and David, (1987) concluded that the biogeography of the Neogene and Recent bryozoans in East Mediterranean (Aegean Sea) and West Mediterranean (Tyrhenian Sea) are paleogeographically united. The newly introduced in this study locality Z138, Jabal Zaltan, Northeastern Sirt Basin, (i.e. locality) is an important addition to the previous distribution of Pouyet and David (1987). 
Miocene bryozoans from Jabal Zaltan, Northeast Sirt basin

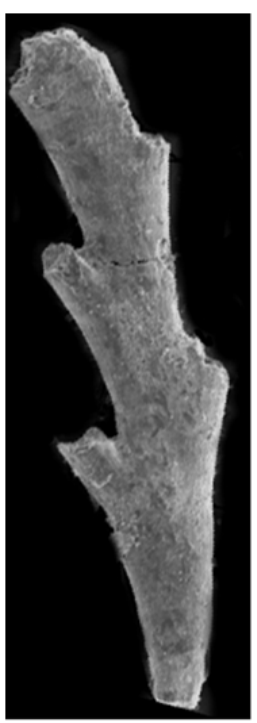

1

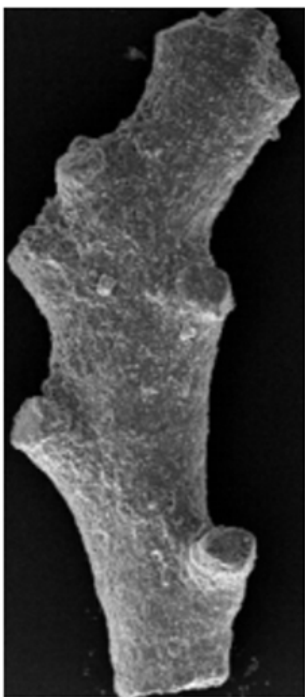

2

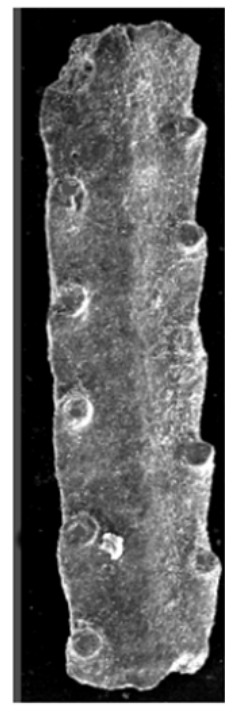

3

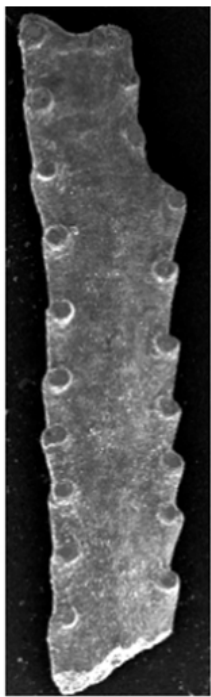

4

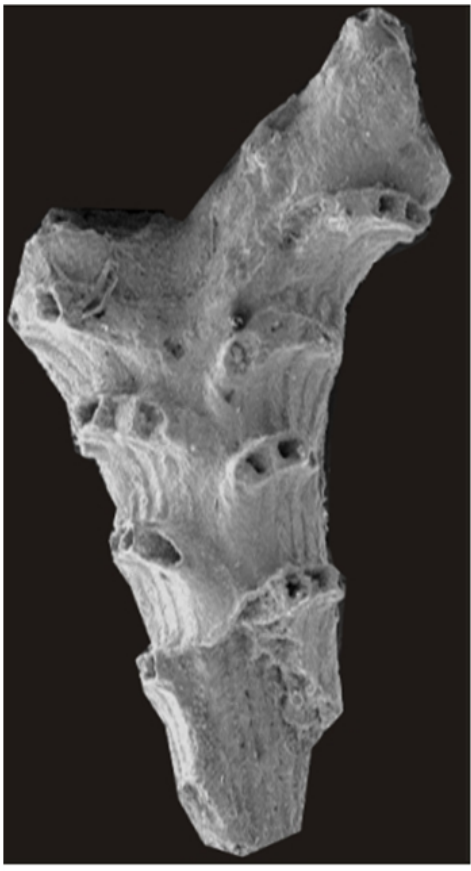

5

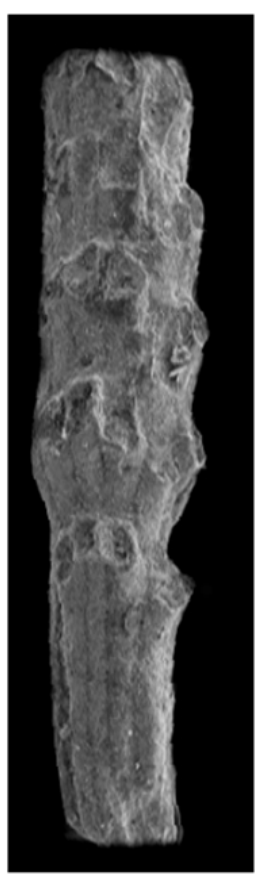

6

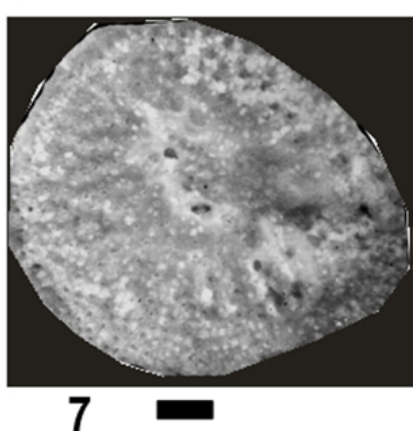

7

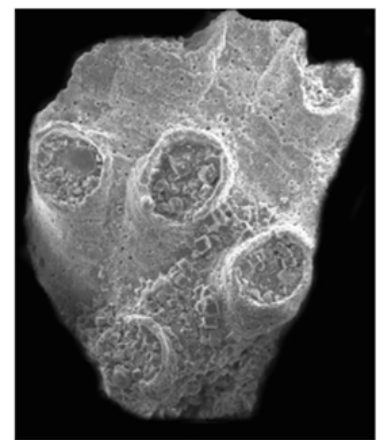

8

Fig. 6: 1. Crisia eburnea (Linnaeus, 1758), 2. Crisia hauri (Reuss, 1848), 3. Crisia hornesi Reuss, 1848, 4. Crisia suezensis El Safori 1994, 5. Exidmonea atlantica (Forbes in Johnston, 1847), 6. Exidmonea undata (Reuss, 1851), 7. Disporella hispida (Fleming, 1828), 8. Ybselosoecia typica (Manzoni, 1878). Scale bar $=200 \mu \mathrm{m}$.

\section{TAPHONOMY}

It is true that bryozoans can be easily mixed with other sediments before the final burial, fact which can obscure some environmental signals. When, however, bryozoans are deposited in situ in life position, they are highly accurate in determining the paleoenvironment. Moreover, the different taphonomical features are very useful in interpreting depositional environments (i.e. bottom current, type of substrate, shell transportation and rate of sedimentation). The abrasion, dissolution, and encrustation are the three taphonomical signs recognized in the investigated assemblages of Ar Rahlah Member of Maradah Formation at Z138 section. 
El-Safori, Y. A. and Muftah, A. M.

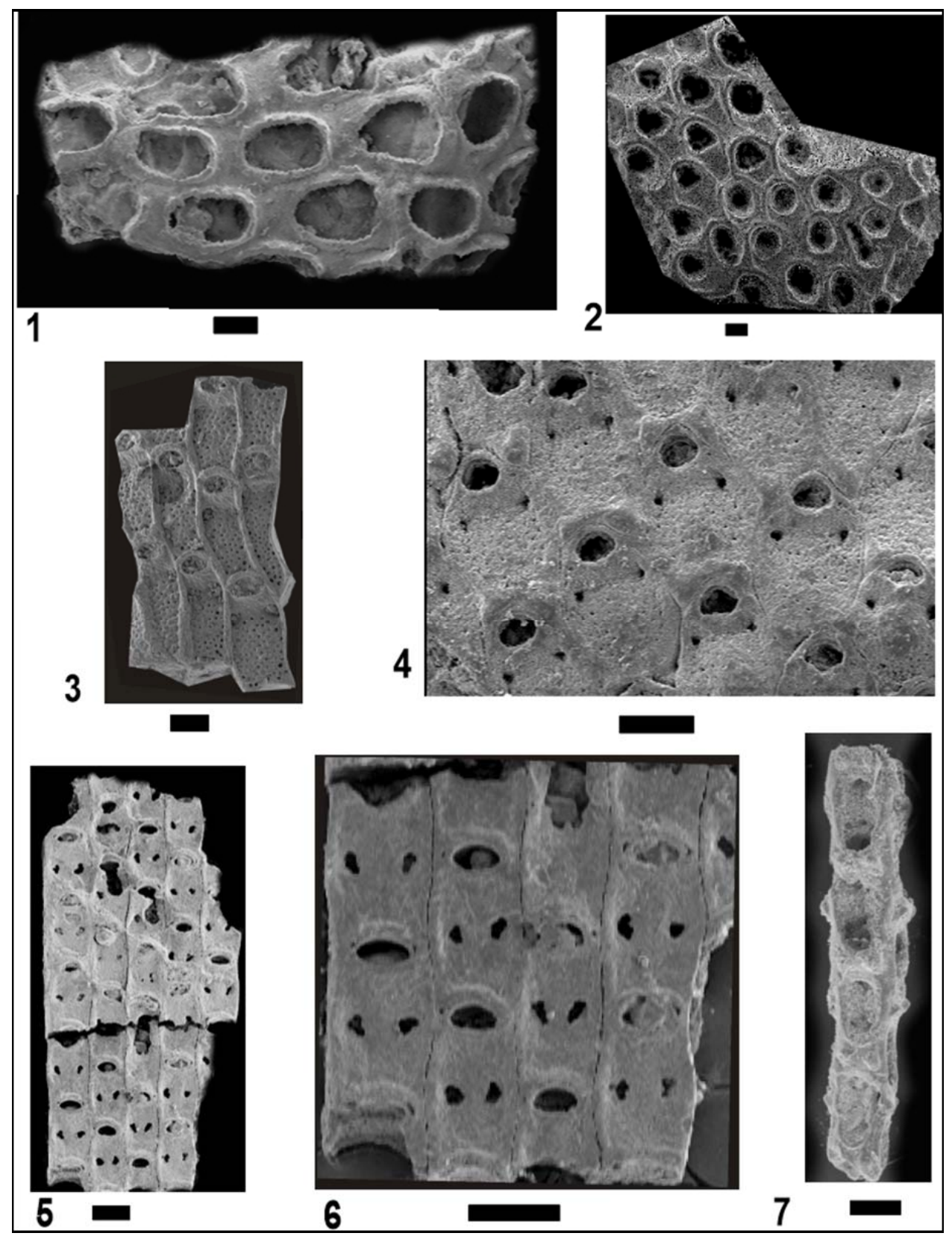

Fig. 7: 1. Conopeuim lacroixi (Audouin 1826), 2. Smittipora sp., 3. Calpensia gracilis (Münster, 1826$), 4$. Calpensia spinosa n. sp., 5, 6. Thalamoporella zaltaniensis n. sp., 7. Nellia tenella (Lamarck, 1816). Scale bar $=200 \mu \mathrm{m}$.

\section{CONCLUSIONS}

The productive carbonate deposits of Ar Rahla Member of Maradah Formation (Serravallian) at the studied locality Z138 in Jabal Zaltan, Sirt Basin yields common bryozoan remains. A descriptive taxonomy has been performed with illustrations for twenty-one species from this measured Serravalian Maradah Formation. The identified taxa consist of $48 \%$ encrusted forms (which are represented by Membraniporiform and Celleporiform) and 52\% erecting forms (which are representing by rigid growthform and erect flexible growth form). Among this unique assemblage only two new species namely Calpensia spinosa n. sp. and Thalamoporella zaltaniensis n. sp. have been described in this paper. Shells of Cubitostrea mostly provide the most attachments of the encrusted bryozoan forms. 
Miocene bryozoans from Jabal Zaltan, Northeast Sirt basin

The reported species are of Mediterranean/Atlantic distributional affinity. However the new species are considered endemic. The limestones and marls are rich in erect flexible and encrusting forms which indicate inter-subtidal $(10-50 \mathrm{~m})$ environments with moderate rate of sedimentation and relatively strong wave actions. Highly agitated environment is specially recognized from the investigated assemblages of the lower part of Maradah Formation (Ar Rahlah Member) as suggested on taphonomical basis.

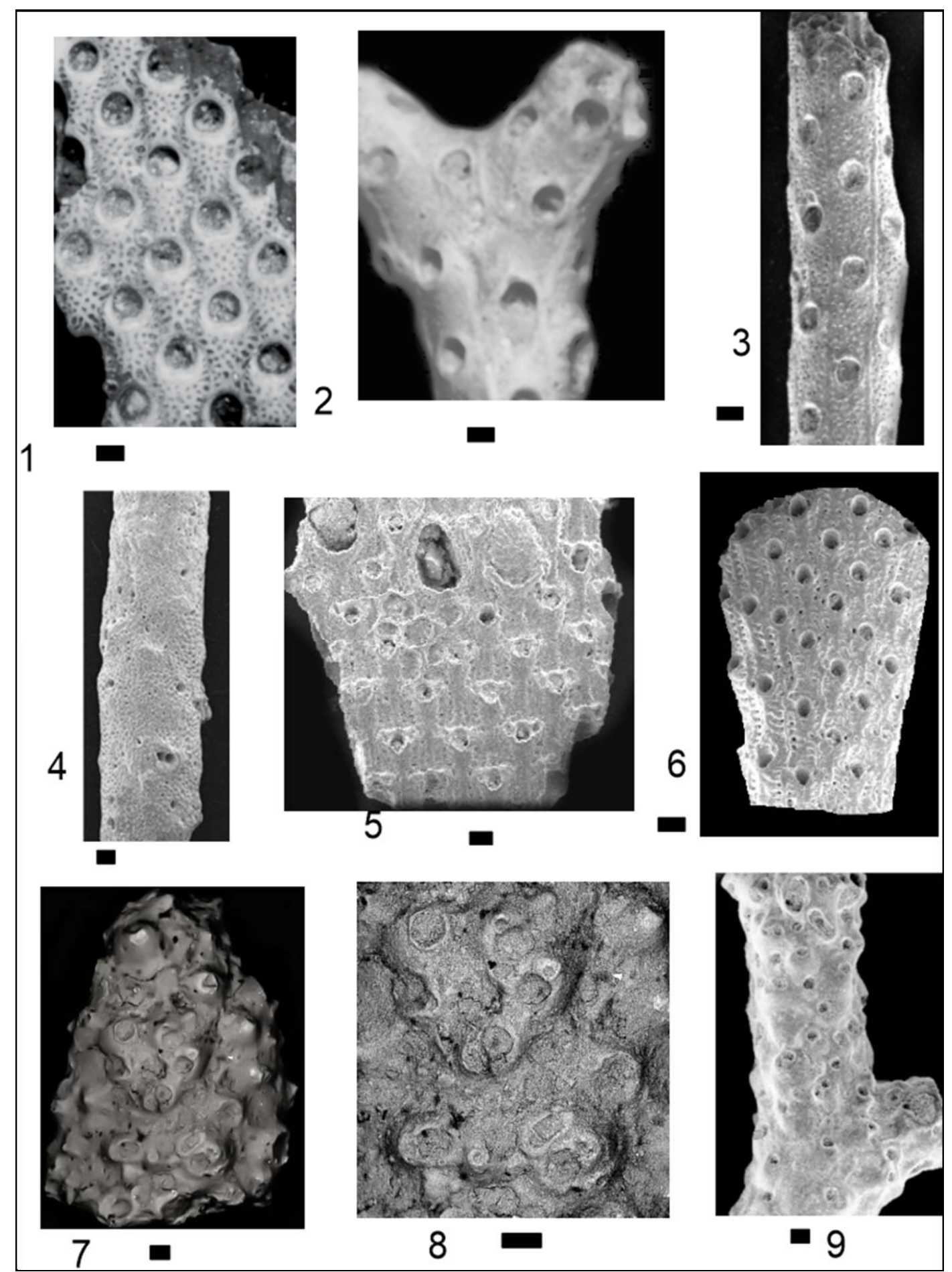

Fig. 8: 1. Mucropetraliella plana Moissette (1988), 2. Dakaria bitubeculata (Canu and lecointre, 1928), 3. Porella cervicornis (Pallas, 1766), 4. Margaretta cereoides (Ellis and Solander, 1786), 5, 6. Metrarabdotos moniliferum (Milne-Edwards, 1836), 7, 8. Celleporina tubilifera Moissette, 1988, 9. Turbicellepora coronopus (Wood, 1844). Scale bar $=200 \mu \mathrm{m}$. 
El-Safori, Y. A. and Muftah, A. M.

Fig. 9: Geographical distribution of main Neogene bryozoans deposits from Mesogean realm (modified after Pouyet and David, 1987).

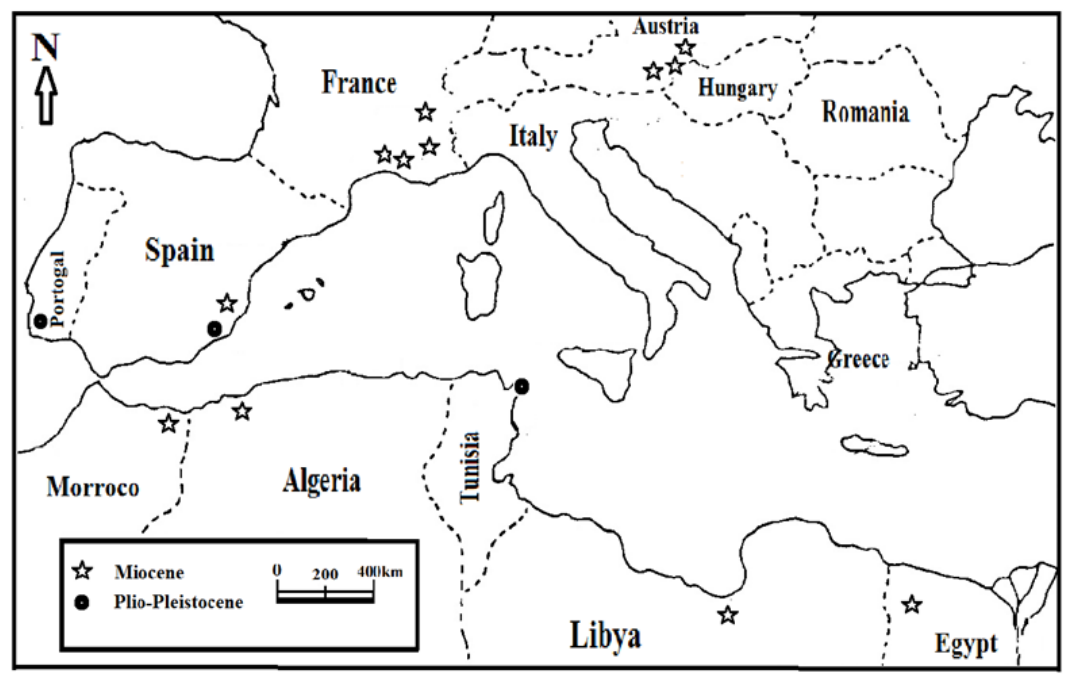

\section{ACKNOWLEDGEMENTS}

We would like to thank East Libya Neogene Research Project (ELNRP) for the logistic support of the field trip. Our gratitude are also to Ain Shams University, Athens University and Benghazi University for giving us all means of support to produce this paper. Special thanks are due to Mr. Walid Al khufaifi and Mr. Faraj Hadia for their scientific and technical help. Also, we are grateful to Professor Dr Adel A. Hegab, Assuit University, and Professor Dr Mohamed S. Ali Minia University for their fruitful discussions of the submitted manuscript.

\section{REFERENCES}

Audouin, V., (1826): Explication sommaire des planches de polypes de l'Egypte et de la Syrie.- In. Déscription de L' Egypt; Hist. Nat., Paris, 1(4), 225-244.

Bassler, R.S., (1953). Bryozoa. In: Moore, R.C. (Ed.) Treatise on Invertabrate Paleontology, Part G; Geol. Soc. America, University of Kansas Press, 175 p.

Bishop, J. D. D. and Hayward, P. J., (1989): SEM Atlas of type and figured material From Robert Lagaaij's. The Pliocene Bryozoa of thelow countries. Meded. Rijks Geol. Dienst, 64, 43-52.

Borg, F., (1926): The stenolaematous Bryozoa Vol. 3. pp.1-1-276. (Bock, S. Further Zoological Results of the Swedish Antarctic Expedition 1901-1903 under the direction of Dr Otto Nordenskjöld) Stockholm, Norstedt and Söner.

Busk, G., (1852): An account of the Polyzoa, and sertularian zoophytes, collected in the Voyage of the Rattlesnake, on the coasts of Australia and the Louisiade Archipelago. In: MacGillivray, J. (editor) Narrative of the Voyage of the H.M.S. Rattlesnake 1, 343-402.

Busk, G. (1854): Catalogue of marine polyzoa in the collection of the British museum, II, British Museum, London, 55-120;

Canu, F. (1912). Etude comparée du Bryozoaires helvétiens de l' Égypt avec les Bryozoaires vivants de la Méditerranée et de La Mer Rouge. Mém. Inst. Egypt., Paris, 6(3), 185-236.

Canu, F. (1918): Etude sur les ovicelles des Bryozoaires cyclostomes (2 ${ }^{\text {nd }}$ contribution). Bulletin de la Societé géologique de France, (4) 17, 345-347.

Canu, F. (1919): Les Bryozoaires fossiles de la région des Corbières. Bulletin de la Societé géologique de France, (4) 18, 294-314

Canu, F. and Bassler, R. S. (1920): North American early Tertiary Bryozoa. Bull. U. S. Nat. Mus., Washington, 106: 1-879.

Canu, F. and Bassler, R. S. (1923): North American later Tertiary and Quaternary Bryozoa. United States National Museum Bulletin, 125, 1-302.

Canu, F. and Lecointer, G. (1928): Les Bryozoaires Cheilostomes des faluns de Touraine et d' Anjou. Mém. Soc. Géol. Francé, N.S. Paris, 4, 5-82. 
Miocene bryozoans from Jabal Zaltan, Northeast Sirt basin

Canu, F. and Bassler, R. S. (1930): Byrozoaires marins de Tunisie. Ann. Stat. Océan. Salambo, Tunis, 1, 91p.

Cheetham, A. H. (1968): Morphology and systematics of the bryozoan genus Metrarabdotos, Smithsonian miscellaneous collections 153, 1-121.

David, L., Mongereau, N., and Pouyet, S. (1972): Bryozoaires du Néogéne du Bassin du Rhône. Gisements burdigaliens de Mus. (Grad). Docum. Lab. Géol. Fac. Sci., Lyon, V. 52: 1-118.

David, L., and Pouyet, S. (1974): Révision des Bryozoaires Cheilostomes Miocénes du Bassin de VienneAutriche. Doc. Un. Lab. Géol. Fac. Sci. Lyon., 60: 83-257.

Desio, A. (1935): Appunti geologici sui dintorni di Sahabi (Sirtica). Rend. R. Ist. Lett., Ser. 2, 68, Fasc. I$\mathrm{V}, 137-144$.

El Hawat, A. S. (1975): Sedimentology and Petrography of the Marada Formation (M. Miocene), Sirte Basin, Libya, Ph. D. thesis, Univ. London, 304p.

El Hawat, A.S. (2008): The pre-Messinian Miocene stratigraphy and sedimentation in the Marada-Zaltan area, Central Sirt Basin, Libya. In: N.T.Boaz, A.El-Arnatui, P.Pavlakis, and M.J.Salem (eds.). CircumMediterranean Geology and Biotic Evolution during the Neogene Period: The Prospective from Libya. University of Garyounis, Benghazi, 23-32.

Ellis, J. and Solander, D. (1786): The natural history of many curious and uncommon zoophytes collected from various parts of the globe. White and Elmsley (Eds.), London, 208p.

El Safori, Y.A. (1994): Stratigraphy and paleontology of some Miocene exposures on the western side of the Gulf of Suez, Egypt, PhD. Thesis, Ain Shams Univ. 278p.

El Safori, Y.A. (2002): Taxonomy, stratigraphy, and paleoecology of the Miocene bryozoans of Siwa Oasis, Egypt, Egypt. Jour. Paleontol., 2, 417-464.

Fleming, J. (1828): A history of British animals. Edinburgh, London, p xxiii, 565 p.

Forbes, A. (1847): A history of the British zoophytes. in Johnston G. (Ed.). Ed. Van Voast, $2^{\text {nd }}$ ed., 1, 1-488.

Gautier, Y.V. (1961): Recherches ecologigues sur les bryozoaires Chilostomes en Mediterranee occidentale. Rec. Trav. Stat. Mar. Endoume, Marseille, 24, 38, 434 p.

Haddadi-Hamdane A. (1996): Bryozoaires du Pliocène du Sahel d'Alger. Documents des Laboratoire de Géologie de Lyon, 140, 1-189.

Harmer, S.F. (1957): The Polyzoa of the Siboga Expedition, Part 4. Cheilostomata Ascophora II. Siboga Expedition Reports 28d: 641-1147

Hayward, P.J., and Ryland, J.S. (1985): Cyclostome bryozoans. In Kermack, D.M. and Barnes, R.S.K. (eds.), Synopses of the British Fauna (new series); Linn. Soc. London: 1-147.

Johnston, G. (1838): A history of British Zoophytes. H. Lizars, Edinburgh, London and Dublin, 1, 1-341.

Johnston, G. (1847): A history of the British zoophytes (John van Voorst; London). 1, xvi, 1-488.

Jullien, J. (1882): Note sur une nouvelle division des Bryozoaires cheilostomiens. Bulletin de la Société Zoologique de France, 6, 271-285.

Jullien, J. (1883): Dragages du 'Travailleur'. Bryozoaires, Espèces draguées dans l'Océan Atlantique en 1881. Bulletin de la Société zoologique de France, 7, 497-529.

Jullien, J. (1888): Sur la sortie et la rentrée du polypide dans les zooecies chez les Bryozoaires Cheilostomiens monodermiés. Bulletin de la Société zoologique de France, 13, 67-68.

Lamarck, J. B. (1816): Histoire naturelle des Animaux sans Vertébres. Paris, Lére édit., 568 p.

Levinsen, G.M.R. (1902): Studies on Bryozoa. Videnskabelige Meddelelser fra den naturhistoriske Foreningi København, 54: 1-31.

Levinsen, G.M.R. (1909): Morphological and Systematic Studies on the Cheilostomatous Bryozoa. Nationale Forfatteres Forlag, Copenhagen, 431p.

Linnaeus, C. (1758): Systema Naturae per regna tria naturae, secundum classes, ordines, genera, species, cum characteribus, differentiis, synonymis, locis. Editio decima, reformata. Laurentius Salvius: Holmiae, ii, $824 \mathrm{p}$.

Manzoni, A. (1878): I briozoi fossili del Miocene d'Austria ed Ungheria. 3 Parte. Crisidea, Idmoneidea, Entalophoridea, Tubuliporidea, Diastoporidea, Cerioporidea. Denkschriften der Kaiserlichen Akademie der Wissenschaften, Wien, 38 (2), 1-24.

Mastera, L. (1985): Geological map of Libya 1:250 000. Sheet Maradah (NH 34-9). Explanatory Booklet, Ind. Res. Cent., Tripoli, $121 \mathrm{p}$. 
El-Safori, Y. A. and Muftah, A. M.

Milne-Edwards, H. (1836): Mémoire sur les Crisies, les Horneres et plusieure autress Polypes vivants ou fossiles dont l'organisation est analogue a celle des Tubulipores. Ann. Sci. Nat., Paris, 2 (9), 193-238.

Milne-Edwards, H. (1838): Mémoire sur les Crisies, les Hornéres et plusieurs autres Polypes vivants ou fossiles dont l'organisation est analogue à celle des Tubulipores. Annales des Sciences naturelles, Zoologie and Biologie animale, (2) 9, 193-238.

Moissette, P., 1988. Faunes de bryozoaires du Messinien d' Algérie occidentale. Doc. Lab. Géol. Lyon, $102,351 \mathrm{p}$.

Münster, G. v. (1826): Bryozoa- in Goldfuss. Petrefactae Germaniae: Abbildungen und Beschreibungen der Petrefacten Deutschlands und der angrenzenden Länder, A.: Petrefacta Germaniae. Arnz and Co., Düsseldorf, 1, 23-41.

d'Orbigny, A. (1852): Paléontologie française. Terrains crétacés - Tome 5: Bryozoaires, 5, 249-264.

Pallas, P.S. (1766): Elenchus zoophytorum, sistens generum adumbr-ations generaliores et specierum Cognitarum succinctas desc-riptions, cum selectis auctorum synonymis. Van Cleef (ed), La Haye, 8, 451p.

Pouyet, S. and David, L. (1979): Revision systematique du genre steginoporella Smitt, 1873. (Bryozoa Cheilostomata). Geobios, Lyon, 12, 6, 763-817.

Pouyet, S. and David, L., (1987): Stratigraphical and biogeographical significance of bryozoan faunas from Miocene to Recent in Tethys and Paratethys. Ann. Inst. Geol. Publ. Hung., Budapest (Proc. $5^{\text {th }}$ RCMNS Congress), 70, 455-462.

Pouyet, S., and Moissette, P. (1992): Bryozaires du Pliocene D'altavilla (Sicile-Italie): Revision de la collection Cipolla, Nouvelles donnees, paleoecologi. Palaeontographica, Stuttgart, No. 223, 19-101.

Reuss, A. E. (1848): Die fossilen polyparien des Wiener Tertiarbeckens. Haiding Naturwiss. Abh., Wien, 2(1), 1-109.

Reuss, A.E. (1851): Ein Beitrag zur Paläontologie der Tertiärschichten von Oberschlesiens. Zeitschrift der Deutsche geologischen Gesellschaft, 3, 149-184.

Reuss, A. E. (1866): Die Foraminiferen, Anthozoen und Bryozoen des deutschen Septarienthones. Ein Beitrag zur Fauna der mitteloligocänen 'l'ertiärschichten. (Denkschr. d. kais. Akad. d. Wissensch. zu Wien. Mathem.-naturw. $\mathrm{Cl}$. Band XXV.)

Schmid, B. (1989): Cheilostome Bryozoen aus dem Badenien (Miozän) von Nussdorf (Wien). Beitr. Paläont. Oesterr., 15, 101p.

Smitt, F.A. (1867): Kritisk förteckning öfver Skandinaviens Hafs-Bryozoer. IV. Öfversigt af Kongliga VetenskapsAkademiens Förhandlingar, 1867, 24(6), 3-230.

Vavra, N., 1974. Cyclostome Bryozoen aus dem Badenien (Mittelmiozän) von baden bei Wien (Niederosterreich). Neues Jahr. Geol. Paläont. Abh, 147 (3), 343-375.

Vavra, N. (1977):. Bryozoa tertiaria In. zapfe, H: Catalogus Fossilium Austriae. Oesterreich. Akad. Wiss., $3,210 \mathrm{p}$.

Vigneaux, M. (1949): Révision des Bryozoaires néogènes du Bassin d'Aquitaine et essai de classification. Mémoires de la Société Géologique de France, 28, 1-153.

Winston, J. E. (1982): Marine bryozoans (Ectoprocta) of the Indian River area (Florida). Bulletin of the American Museum of Natural History, 173, 99-176.

Wood, S.V. (1844): Descriptive catalogue of the Zoophytes from the Crag. Ann. Mag. Nat. Hist., London, $1(13), 10-21$.

Zágoršek, K., (2010): Bryozoa from the Langhian (Miocene) of the Czech Republic. Part I: Geology of the studies sections, systematic decription of the Orders Cyclostomata, Ctenostomata, and "Anascan" Cheilostomata (Suborders Malacostega Levinsen, 1902 and Flustrina Smitt, 1868). Sborník Národního Musea v Praze - Řada B - Př́rodní vědy Acta Musei Nationalis Pragae Series B. Historia Naturalis, 66 (1-2): 3-136.

Ziko, A. and El-Sorogy, A. S. (1995): New Bryozoan records from the Pleistocene coral reefs, Red Sea coast, Egypt. M. E. R. C. Ain Shams Univ., Earth Sci., 9, 80-92.

Ziko, A., Eweda, SH. and El-Khawaga, S. (2010): Middle Miocene cyclostomatous bryozoans from Cyrenaica, North Western Desert, Egypt. Jour. Paleont., 10, 61-106. 
Miocene bryozoans from Jabal Zaltan, Northeast Sirt basin

$$
\begin{aligned}
& \text { جماعيات الميوسين من جبل زلطن، شمال شرق حوض سرت ، ليبيا } \\
& \text { ياسر الصافورى 'و احمد مفتاح' } \\
& \text { ا قسم الجيولوجيا- كلية العلوم- جامعة عين شمس }
\end{aligned}
$$

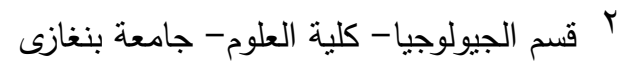

تم تصنيف اثثين وثلاثثن نوع من مستعمرات احافير الجماعيات من تكوين مردة لعصر الميوسين الاوسط (السرافاليان) والمجمعه من قطاع مردة "Z138 "، جبل زلطن، شمال شرق حوض سرت ، ليبيا. من بين هذا التجمع لاحافير الجماعيات تم التعرف على نوعين من المستعمرات المستوطنه وتم دراستهم كنوعين جديدين وهما Calpensia spinosa Thalamoporella zaltaniensis مستعمرات احافير الجماعيات.

تشير الأنواع المسجلة فى تللك الدراسة الى تبعيتها للتوزيعات الحيويه للمتوسطي-الأطلسي فى حين تعتبر الأنواع الجديدة فى الدراسة الحالية من الأنواع المستوطنة. كذذلك توفرت انواع الجماعيات ذات نمطى النمو القشرى السطحى و مرن الانتصاب فى صخور الحجر الجيري والمارل فى الجزء العلوى من تكوين مردة و نتشير تللك المستعمرات الى بيئات تتراوح

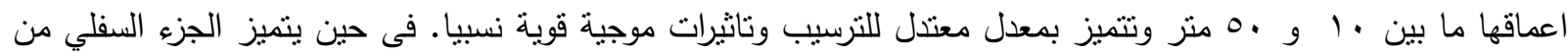
تكوين مردة بالترسيب تحت ثاثيرات موجية قوية كما هو مقترح من زيادة الجماعيات ذات نمط النمو مرن الانتصاب. 